\title{
Magnetic Tunnel Junction based Gradiometer for Detection of Cracks in Cement
}

\author{
Guanyang He ${ }^{1}$, Yiou Zhang ${ }^{1}$, Yuebin Hu ${ }^{1}$, Xixiang Zhang ${ }^{2}$, and Gang Xiao ${ }^{\text {* }}$ \\ ${ }^{1}$ Department of Physics, Brown University, Providence, RI 02912, USA \\ ${ }^{2}$ Physical Science and Engineering Division, King Abdullah University of Science and \\ Technology (KAUST), Thuwal 23955-6900, Saudi Arabia.
}

\begin{abstract}
Magnetic flux leakage (MFL) testing is a widely used technology for detecting structural defects in industry, where new magnetic sensors can provide an enhanced detectability. We have fabricated and developed vortex magnetic tunnel junctions (MTJs) into a new MFL probe, by designing a well-balanced magnetic gradiometer with a base length of $4 \mathrm{~cm}$. Such gradiometer has exhibited a field detectability of $1.6 \mathrm{nT} / \sqrt{\mathrm{Hz}}$ at $1 \mathrm{kHz}$, and a superior common-mode rejection ratio (CMRR) of $82 \mathrm{~dB}$. We have studied multiple configurations of crack detection in weakly magnetic cement by simulations, and provided corresponding experimental confirmations by this MFL probe. It has achieved a large standoff distance of $22 \mathrm{~mm}$, which finds its applications in energy industry and other infrastructural systems.
\end{abstract}

*Author to whom correspondence should be addressed: Gang_Xiao@brown.edu 


\section{Introduction}

Nondestructive testing (NDT) is widely used in industry for noninvasive inspecting materials for defects or inhomogeneity. Common techniques in NDT include ultrasonic sensing, Eddy-current testing, and magnetic flux leakage (MFL) measurement. Eddy-current testing is only effective for conductive metals. ${ }^{1-3}$ The distance between the sensor and the sample surface to be detected, defined as the standoff distance, is typically as small as $1 \mathrm{~mm}$ for Eddy-current testing. ${ }^{4-9}$ Meanwhile, MFL detects the magnetic field leakage in the vicinity of structural defects. Tunneling magneto-resistive (TMR) sensors have been applied in MFL exhibiting $20 \mathrm{~cm}$ standoff distances, although with a relatively low spatial resolution and the restriction to strongly ferromagnetic materials. ${ }^{10}$ For weakly magnetic materials, the decay of magnetic signals over distance limits the standoff distance. In this case, giant magneto-resistive (GMR) sensors have realized a standoff distance of $3 \mathrm{~mm},{ }^{11}$ while for giant magneto-impedance (GMI) effect it is $2 \mathrm{~mm} .{ }^{12}$ The use of planar Hall magneto-resistive sensor has extended the distance to $7 \mathrm{~mm} .{ }^{13}$ While ferrous metals are prevalent in infrastructure, insulating cement can also become weakly magnetic for MFL by impregnating magnetic powders into its slurry (Class $\mathrm{H}$ cement). ${ }^{14}$ However, to inspect the structural integrity of such cement, the limited standoff distance of MFL becomes in conflict with the centimeter-thick fiberglass casing on a cement sheath. ${ }^{15}$ Therefore, in this work we intend to develop a new MFL probe with much extended the standoff distance. In view of the Deepwater Horizon oil spill disaster in 2010 caused by defective cement in boreholes, ${ }^{16}$ our probe can be critically imperative to public safety.

In MFL testing, an excitation coil is used to induce magnetization in the defective material. Compared to sophisticate self-nulling coil configurations, ${ }^{17}$ it is more convenient to use a magnetic gradiometer, which is compact and immune to the excitation field. A gradiometer takes the differential signal from two magnetic sensors separated by certain distance called base length, to distinguish a gradient field from common backgrounds such as the excitation field, the Earth field, and the environmental electromagnetic interference. ${ }^{18}$ It also allows the gradiometer to operate in harsh environment with magnetic interferences, such as in an underground borehole. The gradient field could come from localized magnetic sources such as a crack on the cement, since it breaks the homogeneous magnetization. Various types of magnetic sensors including optical magnetometer, fluxgate, diamond nitrogen-vacancy center magnetometer, and SQUID 
(Superconducting Quantum Interference Device) have been used to construct gradiometers. ${ }^{19-22}$ Nevertheless, they suffer from large physical sizes $(5-10 \mathrm{~cm})$, high power consumption $(100-$ $200 \mathrm{~mW})$, low roll-off frequency $(10-1000 \mathrm{~Hz})$, small dynamic range $(0.5 \mathrm{mT})$, or the need of sophisticate accessories (laser pump to cryogenics). On the other hand, highly sensitive magnetic tunneling junction (MTJ) sensors enjoy compelling advantages, ${ }^{23}$ including low power consumption ( $1 \mathrm{~mW}$ ), broad bandwidth (up to $\mathrm{GHz}$ ), miniaturized size $(\sim 10 \mu \mathrm{m}$ ), and a compatibility with silicon circuit technology. In this work, the novel MFL probe we propose is based on a magnetic gradiometer consisting of state-of-the-art MTJ sensors.

\section{Design of Magnetic Gradiometer}

For the optimal ambient field cancellation, sensitivities of the two magnetic sensors must be precisely matched all the time. Hysteretic sensors are thus not suitable, due to the time drift in sensitivities caused by irreversible magnetic domain wall motions. For this purpose, we have fabricated MTJs with vortex magnetization in the free layer, which can assure non-hysteretic operations. The layer stack is sequenced as: $\mathrm{Si} / \mathrm{SiO}_{2} / \mathrm{Ta}(50) / \mathrm{Ru}(300) / \mathrm{Ta}(50) / \mathrm{Ru}(20) / \mathrm{Ir}_{22} \mathrm{Mn} 78(180)$ $/ \mathrm{Co}_{00} \mathrm{Fe}_{50}(30) / \mathrm{Ru}(8.5) / \mathrm{Co}_{40} \mathrm{Fe}_{40} \mathrm{~B}_{20}(30) / \mathrm{Co}_{00} \mathrm{Fe} 50(5) / \mathrm{MgO}(29) / \mathrm{Co}_{40} \mathrm{Fe}_{40} \mathrm{~B}_{20}(4) / \mathrm{Co}_{0} \mathrm{Fe}_{30}(5) / \mathrm{Co} 40 \mathrm{Fe}$ ${ }_{40} \mathrm{~B}_{20}(600) / \mathrm{Ta}(50) / \mathrm{Ru}(100)$, where the numbers in parentheses represent the thickness of each layer in angstrom. The fabrication process includes magnetron sputtering at a high vacuum of base pressure $2 \times 10^{-8}$ torr, photolithography, ion milling, and thermal annealing at $553 \mathrm{~K}$ for 1 hour. $^{24}$ Each MTJ element has a circular disk shape of $5 \mu \mathrm{m}$ diameter, and $48 \times 32$ such MTJs formed a compact array with a size of $0.5 \times 0.5 \mathrm{~mm}^{2}$, with 48 MTJs connected in series as one row and 32 rows in parallel, shown in the optical micrograph of Fig. 1(a). The performance of such new MTJ sensor is fully characterized in our recent work, ${ }^{25}$ which shows a large linear operation range of $+/-10 \mathrm{mT}$. In this range the sensor is hysteresis-free, with a decent sensitivity of $3.0 \% / \mathrm{mT}$ (the change of magnetoresistance per milli-tesla), which only decreases to $2.7 \% / \mathrm{mT}$ at $+/-10 \mathrm{mT}$. In a wide temperature range from 250 to $350 \mathrm{~K}$, the thermal coefficient of sensitivity is $-400 \mathrm{ppm} / \mathrm{K}$, indicating a high degree of thermal stability. The gradiometer building on this MTJ sensor works well within these ranges. 
Fig. 1(a) shows our gradiometer with two MTJ sensors wire-bonded onto a printed circuit board (PCB), whose signal conditioning circuitry is shown in Fig. 1(c). The circuit is powered by four AAA batteries. Two sensors each under a $1 \mathrm{~V}$ bias voltage are separated by a base length of $4 \mathrm{~cm}$. The voltage signals from two sensors first pass through high-pass filters with $-3 \mathrm{~dB}$ point at $5 \mathrm{~Hz}$, then get boosted separately by two identical amplifiers (INA828) of negligible noise $7 \mathrm{nV} / \sqrt{\mathrm{Hz}}$. The signal from the "signal sensor" at the edge of the PCB is amplified by 50 times, while the other "reference sensor" has a tunable amplification factor between 33 and 100, adjustable through a potentiometer. After an initial adjustment of the potentiometer, a close match of two sensitivities can be achieved and maintained. Next, the amplified signals are differentiated and read by data acquisition devices (DAQ). Software-based lock-in amplification is performed finally to extract the signal at a specific frequency, with a small time constant of $200 \mathrm{~ms}$ ( $0.7 \mathrm{~Hz}$ noise bandwidth).

The voltage output of the gradiometer can be expressed as:

$$
V=D\left[H\left(z_{1}\right)-H\left(z_{2}\right)\right]+C\left[H\left(z_{1}\right)+H\left(z_{2}\right)\right] / 2
$$

where $z_{1}$ and $z_{2}$ are the positions of the signal and reference sensors, $D$ and $C$ are meant to be the sensitivities for gradient and uniform fields, respectively. Calibration for these parameters is done in a pair of single-axis Helmholtz coils. When the current in two coils runs in parallel directions, a uniform magnetic field is generated and $C=H\left(z_{1}\right) / V=H\left(z_{2}\right) / V$ is obtained. When in antiparallel directions, a gradient field gives $D=\left[H\left(z_{1}\right)-H\left(z_{2}\right)\right] / V$. In Fig. 1(b), the voltage signals from the gradiometer in these two kinds of fields are fitted by a linear relation, from which we obtained $D=989.6 \mathrm{~V} / \mathrm{T}$ and $C=0.078 \mathrm{~V} / \mathrm{T}$. It gives a large CMRR (common-mode rejection ratio) of $82 \mathrm{~dB}$, even comparable to a SQUID gradiometer operating at liquid helium temperature. ${ }^{26}$ Moreover, we have measured the intrinsic noise power spectrum density of the gradiometer at no external magnetic field in an unshielded environment, and divided it by the sensitivity $D$ to obtain the magnetic field detectability in Fig. 1(d). At our $1 \mathrm{kHz}$ operating frequency of the gradiometer, the field detectability reaches $1.6 n T / \sqrt{\mathrm{Hz}}$. 


\section{MFL Probe Modelling and Testing}

To demonstrate its actual configuration in the inspection of wellbore integrity, Fig. 2(a) shows a MFL testing scheme we propose inside an oil wellbore, where a testing platform with 8 gradiometers installed in a circumferential configuration are inserted into a wellbore. The platform rotates and scans circumferentially to detect hidden cracks in the cement behind the casing. Considering the large size of the wellbore, close to its inner surface, the rotating scan is analogous to a planar measurement if we neglect the surface curvature, as shown in Fig. 2(b). This represents an elementary defect unit as a benchmark, from which multiple kinds of defects in miscellaneous situations can be estimated.

\subsection{Numerical Simulation of cracked magnetic cement and MFL probe}

Next, we modeled the magnetic cement in simulation as a large flat slab with a thickness of $5 \mathrm{~cm}$, similar to Fig. 2(b). Fig. 3(a) shows its sectional view corresponding to the X-Z plane defined in Fig. 2(b). The signal sensor of the gradiometer is located above the surface with a standoff distance of $h_{1}$. Embedded beneath the surface is a void ("crack") in the shape of a long square tube, placed along the Y-direction with a cross section of $2 \times 2 \mathrm{~cm}^{2}$. Such square-shaped crack is assumed as a general case, since cracks of other irregular shapes can also be detected as long as their sizes are similar. The depth of the crack is $h_{2}$, and the horizontal distance from the crack to the signal sensor is $S$. The excitation coil for magnetization induction has a diameter of $2.5 \mathrm{~cm}$, a $3.4 \mathrm{~cm}$ length, and 120 turns winding, operating with $1 \mathrm{~A}$ current at $1 \mathrm{kHz}$. The gradiometer is placed symmetrically along the axis of the coil, so that the excitation field is common to both signal and reference sensors. The sensors measure the vertical component $H_{Z}$ (Z-axis) of the magnetic fields. Under these conditions, $\mathrm{COMSOL}^{\circledR}$ Multiphysics software is used to simulate the magnetic field lines shown as red arrows in Fig. 3(a), based on Ampere's law. The cell size used for meshing is $0.05 \mathrm{~cm}$ near the sensor region, and the zero-potential boundary is a spherical surface 2 meters away from the sensors. The relative permeability of the cement is taken as 1.09 , which will be explained in the later experimental section. From the simulation, the common-mode field at the sensor position is $1.14 \mathrm{mT}$ within the MTJ linear range. The spatially differential field $\Delta H_{Z}$ due to the embedded "crack" is sensed by the dual signal and reference sensor, corresponding to the gradiometer output. 
Fig. 3(b) shows the simulated map of the differential field $\Delta H_{Z}$ in the plane of Fig. 3(a), at positions of different $S$ and $h_{1}$, with $h_{2}=0$ as a surface crack case. Fig. 3(c) shows an exponential decay of $\Delta H_{Z}$ at $\mathrm{S}=0$ position of Fig. 3(b), with a fitting line on the simulation data. During an MFL measurement, the gradiometer scans horizontally across the crack region. Accordingly, Fig. 3(d) shows $\Delta H_{Z}$ as a function of $S$, mimicking scans under different $h_{1}, h_{2}$ values (in cm), with values at $S= \pm 6 \mathrm{~cm}$ deducted as offset. For $h_{1}+h_{2}=2$, considering the cases of a surface crack $\left(h_{1}=2, h_{2}\right.$ $=0)$ and an embedded crack $\left(h_{1}=1, h_{2}=1\right)$, the scanned $\Delta H_{Z}(S)$ profiles resembles a similar bell curve with a peak value of $860 \mathrm{nT}$ and a FWHM (full width at half maximum) of about $3.2 \mathrm{~cm}$. Increasing $h_{1}+h_{2}$ to $3 \mathrm{~cm}$, we examined three cases: a surface crack $\left(h_{1}=3, h_{2}=0\right)$, a shallow crack $\left(h_{1}=2, h_{2}=1\right)$, and a deep crack $\left(h_{1}=1, h_{2}=2\right)$. Again, all the scanned $\Delta H_{Z}(\mathrm{~S})$ profiles are similar, with a peak value of $210 \mathrm{nT}$ and a FWHM of about $4.2 \mathrm{~cm}$. Conclusively speaking, regardless of how deep the crack is imbedded, its $\Delta H_{Z}$ decays exponentially with the sensor-tocrack distance $h_{1}+h_{2}$. Considering a $300 \mathrm{nT}$ detectable limit which is our experimental result to be presented later, the intercept on the fitting line in Fig. 3(c) implies that the gradiometer could see such surface crack with standoff distances between 2 and $3 \mathrm{~cm}$. The characteristic FWHM is further studied in Fig. 3(e) for a smaller coil of $1.25 \mathrm{~cm}$ diameter and a smaller crack with cross section of $0.5 \times 0.5 \mathrm{~cm}^{2}$, with other conditions the same as the $\left(h_{1}=2, h_{2}=0\right)$ case in Fig. 3(d). FWHM decreases from 3.2 to $2.2 \mathrm{~cm}$ reasonably for a smaller crack. With a smaller coil the FWHM doesn't change, but the amplitude of $\Delta H_{Z}$ decreases. This is on account of a more localized field, which magnetized less cement region.

\subsection{MFL Probe Testing}

Finally, we proceed to test the gradiometer-based MFL probe on real magnetic cement for experimental confirmation. Fig. 4(a) shows the experimental setup for MFL testing using our gradiometer, as an identical realization of Fig. 2(b). The surface crack $\left(h_{2}=0\right)$ case is imitated by embedding a square-shaped plastic rod into the magnetic cement. The magnetic cement is imitated by impregnating a small amount of ferromagnetic $\mathrm{Fe}_{3} \mathrm{O}_{4}$ particle powder (Alpha Chemicals ${ }^{\circledR}$ ) into common plaster. The volume fraction of the $\mathrm{Fe}_{3} \mathrm{O}_{4}$ particles is $4.6 \%$ corresponding to a mass 
fraction of $6.0 \%$. Fig. 4(b) shows its magnetic hysteresis loop at room temperature, measured by the vibrating sample magnetometer in the Quantum Design ${ }^{\circledR}$ Physical Property Measurement System. The central slope of the magnetic hysteresis curve gives a relative permeability of 1.09 for the magnetic cement. Relevant works in literature typically use similar volume fractions of magnetic particles in cement (4\%), larger values of cement permeability (above 1.25) or wellbore structures with big cracks (10 to $20 \mathrm{~cm})^{15}$, and the iron powder in the impregnated cement has shown negligible impact on overall mechanical behavior, ${ }^{14}$ Comparatively, our experiments focus on smaller crack sizes $(2 \mathrm{~cm})$ and a more available permeability (1.09). Fig. 4(c) shows the voltage output of the gradiometer in a scan across the crack. At each "S" position the voltage signal will be amplified and read by the lock-in amplifier for 5 seconds. In this period of time, the mean value is recorded as the voltage output, and the standard deviation is recorded as the noise signal (error bar in the figure). The rather long averaging time is to ensure an accurate determination of the signal level and particularly the noise level. To ensure a good signal-to-noise ratio, the scanning device should remain stabilized during the data collection with a constant standoff distance. The experimental voltage output values on the left axis is converted to a corresponding $\Delta H_{Z}$ on the right axis by previous sensitivity $D=989.6 \mathrm{~V} / \mathrm{T}$. A $300 \mu \mathrm{V}$ voltage fluctuation is observed and plotted as error bars, corresponding to a detectable field limit of $\Delta H_{Z}=300 \mathrm{nT}$. A discernible signal change is observed when the probe moves directly above the crack, demonstrating that the probe is capable of detecting a $2 \mathrm{~cm}$ wide surface crack from around $22 \mathrm{~mm}$ high above. Above this distance, the signal change due to the crack becomes smaller than the noise fluctuation and indiscernible. This is consistent with the simulation prediction in the previous paragraph. The $\Delta H_{Z}$ range in Fig. 4(c) seems bigger than Fig. 3(d), because the latter focuses on larger liftoff distances. To test the response speed of the gradiometer for application considerations, we have quickly moved the gradiometer across the crack at a speed of $2 \mathrm{~cm} / \mathrm{s}$, with the output signal recorded continuously in Fig. 4(d). The presence of the crack is still visible at all distances, yet the peak height is smaller than that of the static measurement with time averaging. This effect is technically due to the large time constant of the lock-in amplification which limits the signal slew rate. 


\section{Discussion}

To further improve the detectable limit, the signal can be enhanced by increasing the relative permeability of the magnetic cement, or generating stronger excitation field. Noticing that with the excitation field and the lock-in amplifier, the $300 \mathrm{nT}$ fluctuation observed in MFL experiment is significantly larger than the gradiometer detectability $1.6 \mathrm{nT} / \sqrt{\mathrm{Hz}}$ at $1 \mathrm{kHz}$ from Fig. 1(d), considering a $1 \mathrm{~Hz}$ bandwidth of our DAQ. One possible origin of the extra noise is the electromagnetic interference from the excitation coil to the gradiometer at the excitation frequency, which may be suppressed by better circuitry isolation. Also, the large current in the excitation coil could fluctuates due to Joule heating. If the extra noise can be eliminated by better equipment design, the detection limit could reach $1.6 \mathrm{nT}$. Such detection limit in the intercept of the fitting in Fig. 3(c) implies an enhanced standoff distance of $7 \mathrm{~cm}$.

In this paper, only a single rectangular crack in the magnetic cement has been modelled and measured, while crack shape may be irregular and/or multiple cracks may be present in a more realistic scenario. Due to the weak paramagnetic nature of the magnetic cement, the excitation magnetic field is only slightly distorted near the crack. As illustrated in Fig. 3(a), the magnetic flux lines do not show any noticeable change with the presence of the crack. This implies that the MFL signal is more sensitive to the volume of the crack, rather than its specific geometry. Hence the generality of a rectangular-shaped crack can be justified. Still, cracks with particular shapes may have significantly enhanced or suppressed MFL signal. Further work includes both numerical simulations and actual measurements on cracks with different shapes, which is beyond the scope of this paper. The ability to distinguish multiple cracks mostly depends on the spatial resolution of the probe. As for our MFL probe, the spatial resolution is mostly limited by the size of the excitation coil. As shown in Fig. 4(e) and discussed in previous section, simply reducing the coil size would only slightly improve the FWHM. In order to have high spatial resolution, both coil size and standoff distance need to be reduced, so that the excitation field is more localized. In situation where exact size and/or number of cracks are more important while high standoff distance is not required, smaller excitation coil would be more suitable. 


\section{Conclusion}

In summary, we have built up a new portable gradiometer based on vortex MTJ sensors, with a 4 $\mathrm{cm}$ base length and a good detectability of $1.6 \mathrm{nT} / \sqrt{\mathrm{Hz}}$ at $1 \mathrm{kHz}$. The gradiometer can be precisely balanced due to the superior stability of its MTJ sensors, leading to an ultra-high CMRR of $82 \mathrm{~dB}$. We have developed an MFL probe with this gradiometer, whose utility is demonstrated in inspecting centimeter-sized cracks on weakly magnetic cement, with a large standoff distance of $22 \mathrm{~mm}$. Every experimental parameter in the demonstration is based on practical industrial considerations, as this probe is designed to provide a ready solution for the wellbore inspection in oil exploitation industry. Besides, multiple configurations for crack detection are explored in our simulations, to provide guidance for general MFL testing designs.

\section{Acknowledgement}

This work was supported by King Abdullah University of Science and Technology (KAUST) through the Sensor Initiative. We thank colleagues at KAUST for discussion and collaboration. Y.Z. acknowledges support from Fermilab-Graduate Instrumentation Research Award from DOE grant number DE-AC05-00OR22725. We used the Heidelberg MLA150 Maskless Aligner, which was under the support of a National Science Foundation Grant No. DMR-1827453. 


\section{References}

B. Wu, Y. J. Wang, X. C. Liu, and C. F. He, Smart Mater Struct 24 (7), 075007 (2015).

J. García-Martín, J. Gómez-Gil, and E. Vázquez-Sánchez, Sensors 11 (3), 2525 (2011).

X. C. Liu, Y. J. Wang, B. Wu, Z. Gao, and C. F. He, J. Sens. 2016, 1 (2016).

K. Tsukada, Y. Haga, K. Morita, N. Song, K. Sakai, T. Kiwa, and W. Y. Cheng, IEEE T MAGN 52 (7), 1 (2016).

D. J. Sadler and C. H. Ahn, Sens. Actuator A Phys. 91 (3), 340 (2001).

T. Dogaru and S. T. Smith, IEEE T MAGN 37 (5), 3831 (2001).

L. S. Rosado, F. A. Cardoso, S. Cardoso, P. M. Ramos, P. P. Freitas, and M. Piedade, Sens. Actuator A Phys. 212, 58 (2014).

A. Jander, C. Smith, and R. Schneider, Magnetoresistive sensors for nondestructive evaluation. (SPIE, 2005).

C. Ye, Y. Wang, M. Wang, L. Udpa, and S. Udpa, NDT \& E International, 102284 (2020). M. A. I. Mohd Noor Sam, Z. Jin, M. Oogane, and Y. Ando, Sensors 19 (21), 4718 (2019).

W. S. Singh, B. Rao, S. Vaidyanathan, T. Jayakumar, and B. Raj, Measurement Science and Technology 19 (1), 015702 (2007).

M. M. Tehranchi, M. Ranjbaran, and H. Eftekhari, Sensors and Actuators A: Physical 170 (1-2), 55 (2011).

H. Q. Pham, B. V. Tran, D. T. Doan, Q. N. Pham, K. Kim, C. Kim, F. Terki, and Q. H. Tran, IEEE T MAGN 54 (6), 1 (2018).

S. D. Nair, T. Patzek, and E. van Oort, (2017).

T. Eltsov and T. W. Patzek, Energies 12 (21), 4211 (2019).

C. Vipulanandan and A. Mohammed, Smart Mater Struct 24 (12), 125020 (2015).

M. Wang, X. Tao, L. Peng, and C. Ye, IEEE Sensors Journal (2020).

C. Affolderbach, M. Stähler, S. Knappe, and R. Wynands, Appl. Phys. B 75 (6-7), 605 (2002).

S. Blakley, I. Fedotov, S. Y. Kilin, and A. Zheltikov, Opt. Lett. 40 (16), 3727 (2015).

A. Sunderland, A. V. Veryaskin, W. McRae, L. Ju, and D. G. Blair, Sens. Actuator A Phys. 147 (2), 529 (2008).

M. Janošek, A. Platil, and J. Vyhnánek, IEEE T MAGN 51 (1), 1 (2015).

M. E. Huber, N. C. Koshnick, H. Bluhm, L. J. Archuleta, T. Azua, P. G. Björnsson, B. W. Gardner, S. T. Halloran, E. A. Lucero, and K. A. Moler, Rev. Sci. Instrum. 79 (5), 053704 (2008).

G. Xiao, in Handbook of Spin Transport and Magnetism (2012), pp. pp 665.

G. He, Y. Zhang, L. Qian, G. Xiao, Q. Zhang, J. C. Santamarina, T. W. Patzek, and X. Zhang, Appl. Phys. Lett. 113 (24), 242401 (2018).

G. He, Y. Zhang, and G. Xiao, Physical Review Applied 14 (3), 034051 (2020).

H. G. Meyer, R. Stolz, A. Chwala, and M. Schulz, Phys. Status Solidi C 2 (5), 1504 (2005). 


\section{Figures and Captions:}
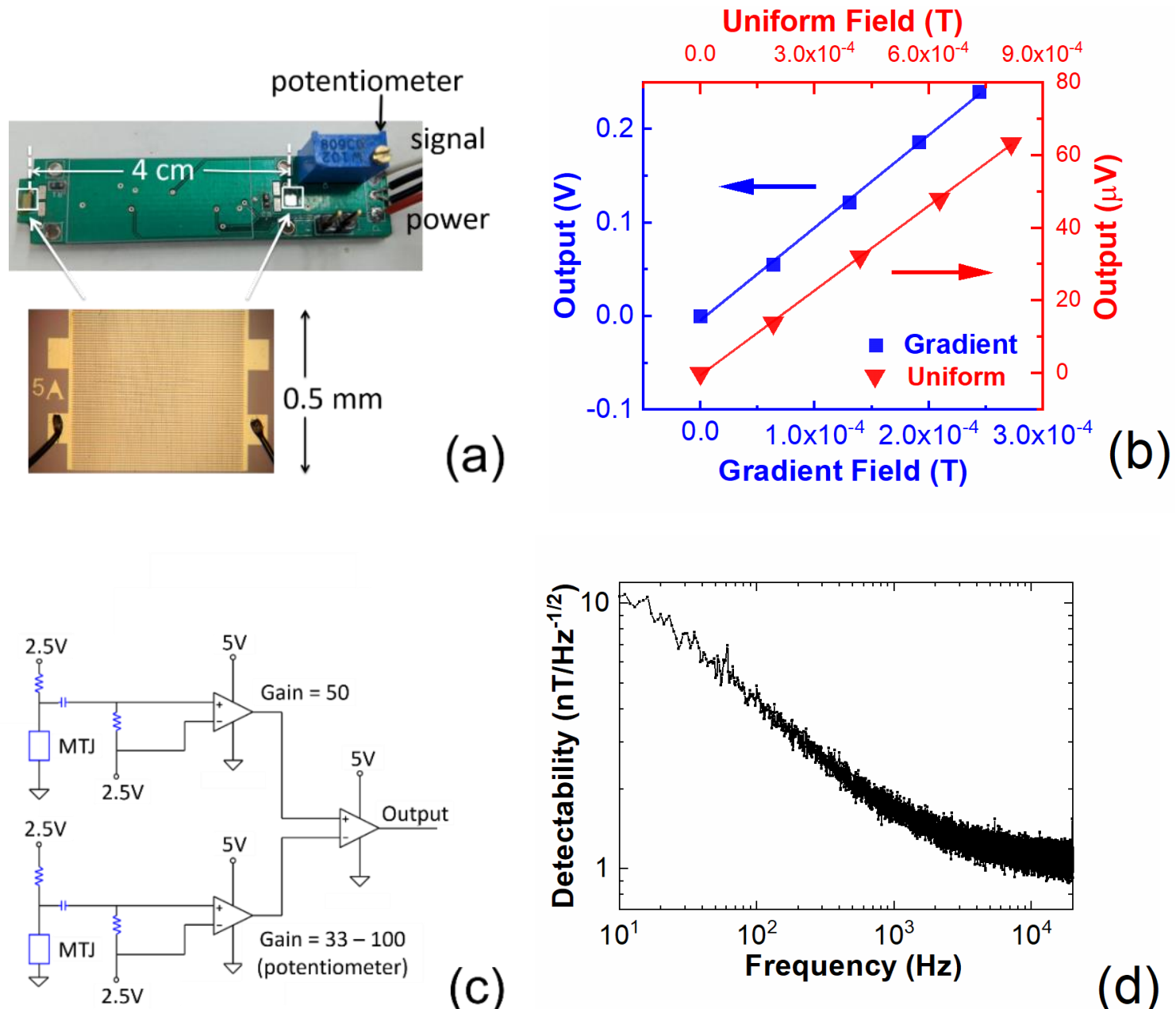

(c)

(d)

Figure 1. (a) Magnetic gradiometer and a micrograph of the MTJ array sensors. Two sensors are mounted at a distance of $4 \mathrm{~cm}$ apart, with one the signal sensor on the left edge of the gradiometer. (b) Voltage output signals of the gradiometer in the Helmholtz coil calibration, with a linear fitting giving the gradient and uniform field sensitivities. (c) Electronic circuit design of the gradiometer. (d) Field detectability of the gradiometer derived from its noise spectrum density. 
(a)

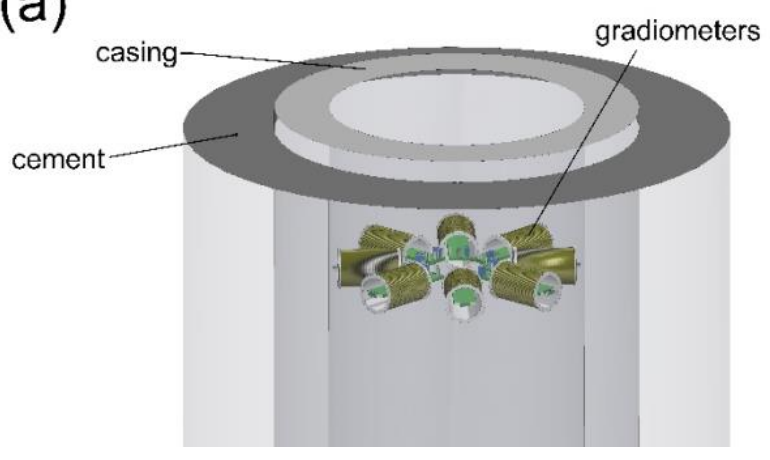

(b)

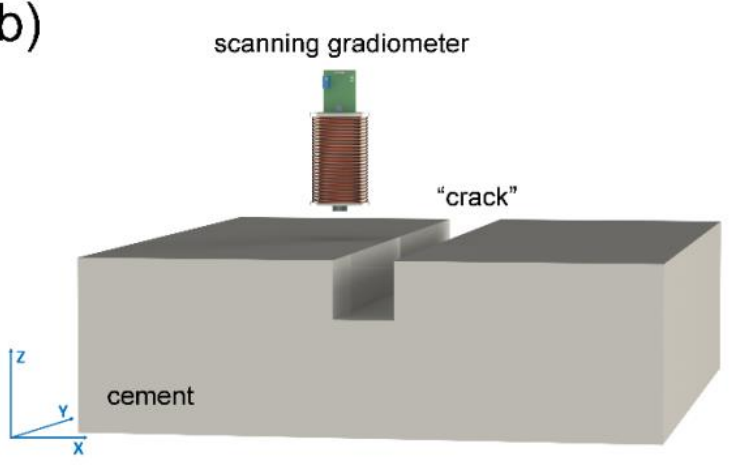

Figure 2. (a) Schematic of a testing platform with 8 gradiometers installed, scanning the oil wellbore circumferentially for cracks in the cement behind the casing. (b) Circumferential scanning in (a) is analogous to a planar scan above a defective cement surface, under the condition that the gradiometer size is negligible to the diameter of the wellbore. 
(a)

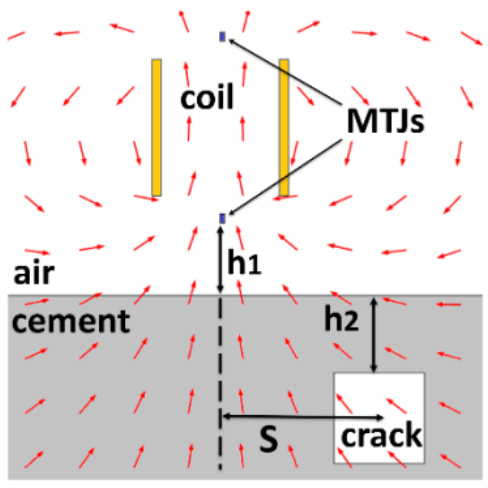

(c)

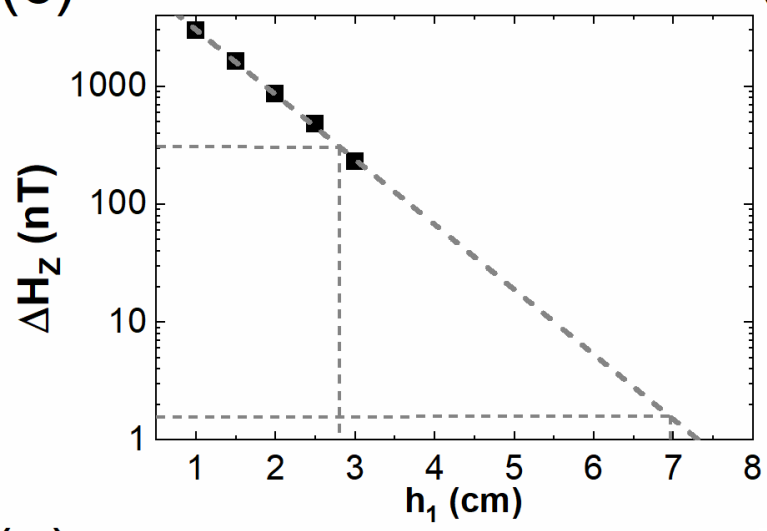

(e)

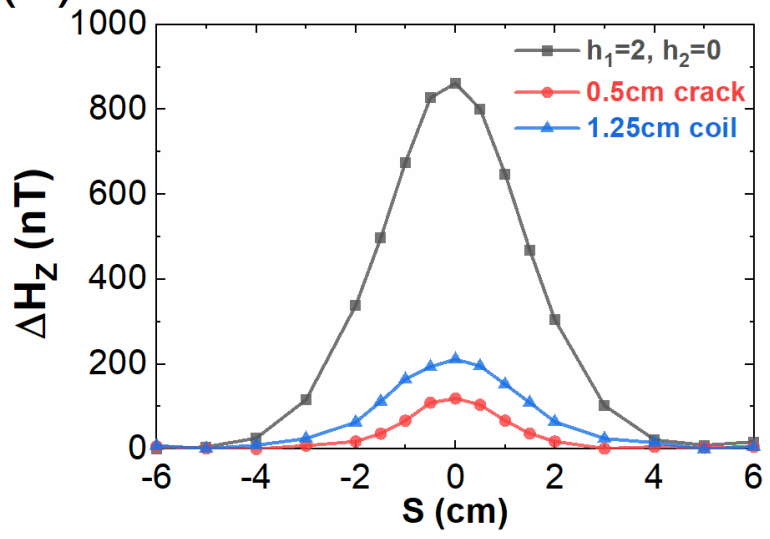

(b)

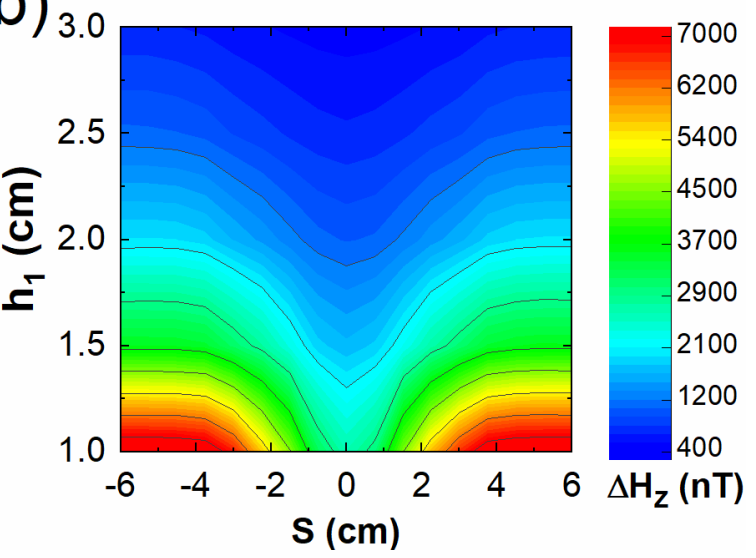

(d)

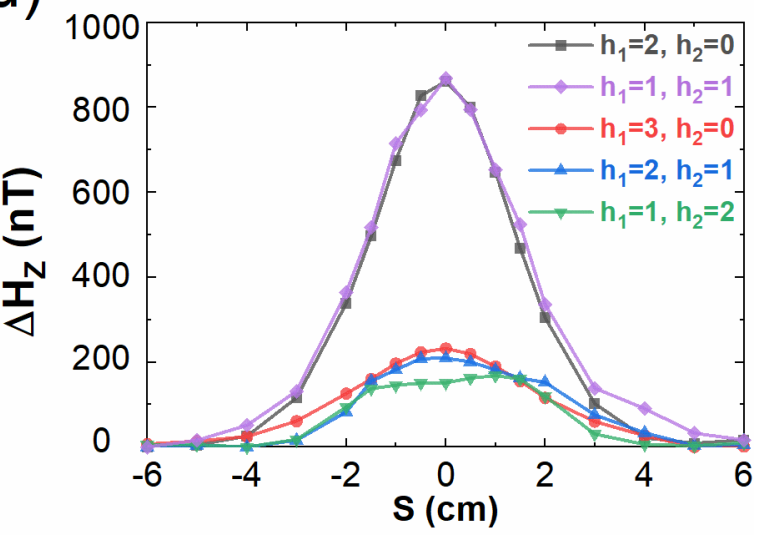

Figure 3. (a) A cross-section view of the X-Z plane of Fig. 2(b), with a square-shaped void defect in the cement, the excitation coil, and the two sensors of the gradiometer. $h_{1}, h_{2}$, and $S$ are defined in the sketch and red arrows are the simulated magnetic induction lines. (b) Simulated $\Delta H_{Z}\left(S, h_{1}\right)$ map in the plane of (a), for $h_{2}=0 \mathrm{~cm}$. (c) The exponential decay of $\Delta H_{Z}\left(0, h_{1}\right)$ over $h_{1}$, at the $\mathrm{S}=$ 
0 position of (b), with a linear fitting. The standoff distances $h_{1}$ corresponding to two detectable limits $\Delta H_{Z}=300 n T$ and $1.6 n T$ are shown by dashed guide lines. (d) $\Delta H_{Z}(S)$ scanned at multiple $\left(h_{1}+h_{2}\right)$ distances to the crack, under the same excitation field. (e) $\Delta H_{Z}(S)$ for a smaller coil or crack, in comparison with the $\mathrm{h}_{1}=2, \mathrm{~h}_{2}=0$ case in (d). 
(a)
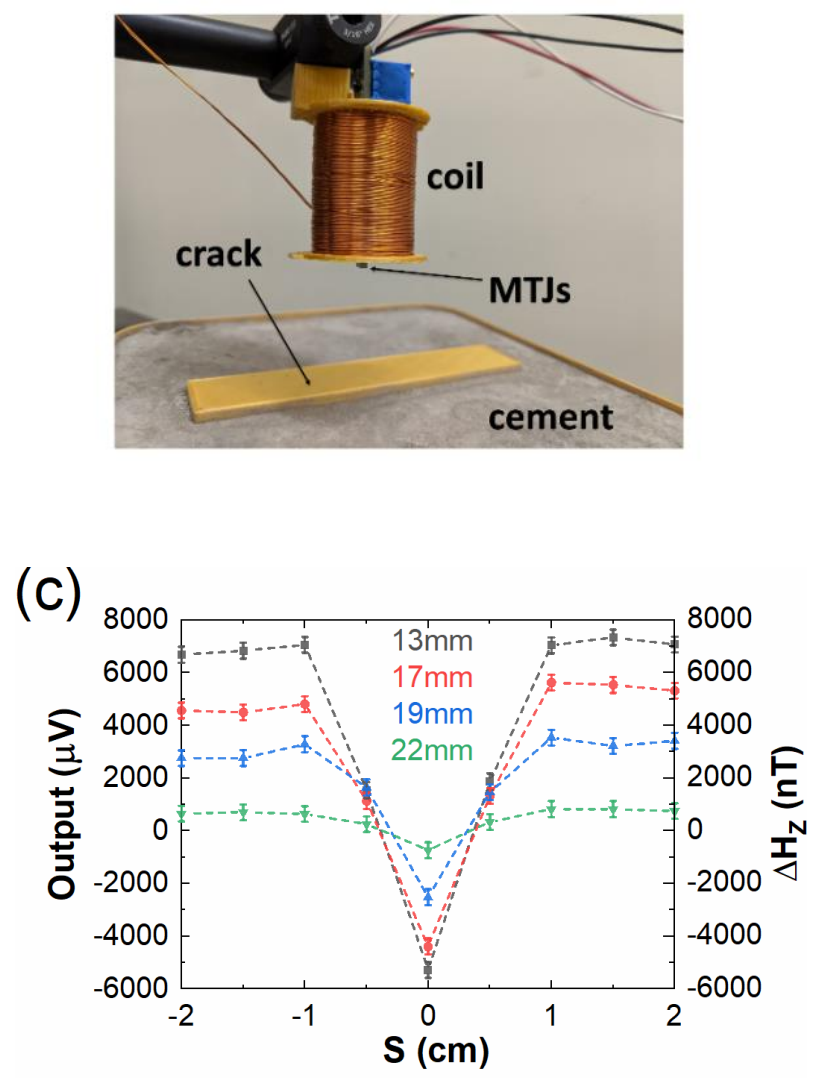
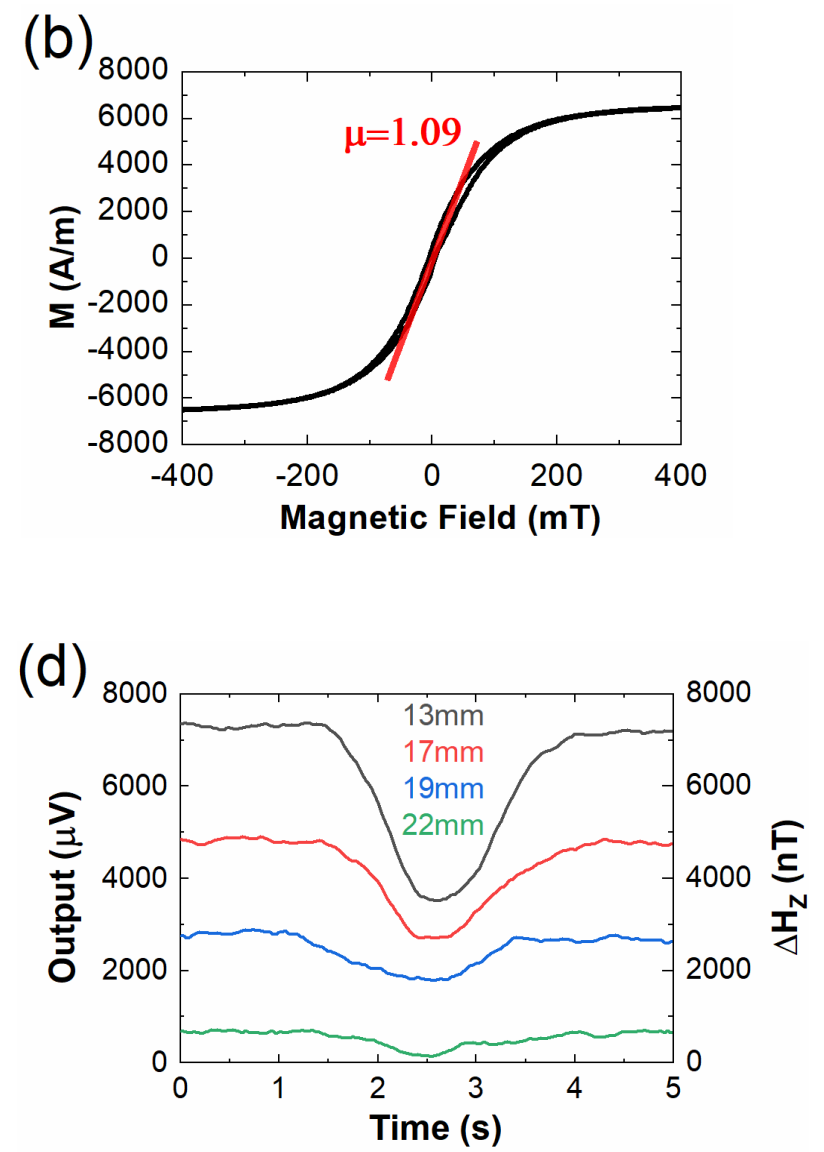

Figure 4. (a) Experimental setup to detect an artificial crack in the cement, using the gradiometer enclosed by the excitation coil. (b) Magnetic hysteresis loop of our magnetic cement impregnated with ferromagnetic $\mathrm{Fe}_{3} \mathrm{O}_{4}$ particles at room temperature. (c) The experimental voltage output of the gradiometer with 5-second averaging (left axis), and the deduced magnetic field $\Delta H_{Z}$ generated by the "crack" (right axis). (d) Similar results to (c), measured in a continuous scan at a $2 \mathrm{~cm} / \mathrm{s}$ scanning speed without time averaging. In (c) and (d), the gradiometer scans at 13, 17, 19, $22 \mathrm{~mm}$ standoff distance above the "crack", and $\Delta H_{Z}$ is calculated by the sensitivity $D=989.6 \mathrm{~V} / T$. 